\title{
Mentorielle Betreuung im Web - Konzepte und Perspektiven für das Fernstudium
}

\author{
Claudia de Witt, Thomas Czerwionka und Sandro Mengel
}

\begin{abstract}
Im vorliegenden Artikel werden zunächst verschiedene für die Betreuung Fernstudierender relevante Konzepte vorgestellt. Nach einem zusammenfassenden Vergleich, der die jeweils unterschiedlichen Rollen der Betreuenden aufzeigt und in eine Unterscheidung zwischen statischen und dynamischen Betreuungskonzepten mündet, werden unter Einbeziehung weiterer Studien zum computergestützten und kollaborativen Lernen Perspektiven für zukünftige Betreuungsszenarien im Fernstudium formuliert. Zentrale Aspekte sind dabei die Gestaltung von Mentoriaten, der drei Basiskonzepte zugrunde liegen sollten (Blended Learning, kooperatives Lernen, diskursbezogene Betreuung), die Gestaltung der Zusammenarbeit der Betreuenden, für die verschiedene Möglichkeiten der Förderung von Kommunikation und Kooperation aufgezeigt werden, und die zu berücksichtigenden Anforderungen an Mentoren/-innen, die vor allem eine umfassende Medienkompetenz und sozial-kommunikative Kompetenz betreffen. Empfohlen wird darüber hinaus die Einrichtung einer koordinierenden Instanz in Form einer/eines übergreifend agierenden Mitarbeiterin/Mitarbeiters für die Betreuungsorganisation (Betreuungskoordinator/in).
\end{abstract}

\section{Einleitung}

Im Fernstudium war und ist die mentorielle Betreuung immer ein wichtiger Erfolgs- und Motivationsfaktor gewesen. So hat sich z. B. die FernUniversität in Hagen seit ihrer Entstehung mit der Notwendigkeit mentorieller Betreuung auseinandergesetzt, Konzepte dafür entwickelt, umgesetzt und evaluiert. Mit dem stärkeren Einsatz Neuer Medien im Fernstudium aber verändern sich auch die Aufgaben, Methoden und die Organisation mentorieller Betreuung.

Betreuung ist jedoch nicht nur für die Fernlehre, sondern auch für die Präsenzuniversitäten, die zunehmend E-Learning einsetzen, ein wichtiger didaktischer Faktor. Bei allen Diskussionen um neue Content- oder Learningmanagementsysteme, Web 2.0 oder Social Software spielt die didaktische Unterstützung beim Lehren und Lernen mit Neuen Medien immer eine grosse Rolle. Auch wenn beim E-Learning von selbstgesteuertem Lernen die Rede und der Lernende dafür verantwortlich ist, den eigenen Lernprozess zu kontrollieren und zu steuern, ist der Lehrende dafür verantwortlich, die entsprechende Unterstützung zu bieten. Und auch bei erwachsenen Lernenden kann selbstgesteuertes Lernen nicht 
von vornherein vorausgesetzt werden. Betreuung im Sinne von Unterstützung ist ein wesentliches didaktisches Element in Lehr- und Lernprozessen. Dafür sind (medien-)didaktische Entscheidungen zu treffen, die z.B. den Anteil an realer und virtueller Betreuung festlegen.

Der vorliegende Artikel, der im Wesentlichen aus einem Projekt zur mentoriellen Betreuung an der FernUniversität in Hagen hervorgegangen ist (vgl. de Witt/Czerwionka/Mengel 2006), soll Perspektiven für die mögliche Ausgestaltung mentorieller Betreuung im Fernstudium aufzeigen. Dazu werden verschiedene Betreuungskonzepte aus dem Bereich des E-Learning beschrieben und miteinander verglichen und unter Einbeziehung weiterer Studien zum computergestützten und kollaborativen Lernen entsprechende Empfehlungen abgeleitet.

\section{Betreuungskonzepte im Vergleich}

In den meisten virtuellen Bildungsangeboten ist eine Betreuung vorgesehen (vgl. Kerres 2001). Im Folgenden werden vier verschiedene Betreuungskonzepte, die in den letzten Jahren in nationalen und internationalen Bildungsprozessen angewendet wurden, vorgestellt und miteinander verglichen. Obwohl sich die Konzepte im Hinblick auf die Rollen und Aufgaben der Betreuenden z. T. deutlich voneinander unterscheiden, sprechen sich die Autoren/-innen aufgrund der gesammelten positiven Erfahrungen für das jeweils von ihnen beschriebene Konzept aus.

\section{Emder Konzept}

Als «ideal empfundenes mentorielles Betreuungskonzept» (Thomaschewski 2005, S. 45) beschreibt Prof. Dr. Jörg Thomaschewski (Fachhochschule Oldenburg/Ostfriesland/Wilhelmshaven) das sogenannte "Emder Konzept», das im Rahmen des Bundesleitprojekts «Virtuelle Fachhochschule» umgesetzt wurde. Auf der Grundlage mehrjähriger Erfahrungen mit Online-Studiengängen benennt Thomaschewski zunächst die für eine erfolgreiche Betreuung wichtigen Randbedingungen eines Online-Studiums. Im Mittelpunkt steht dabei der Informationsfluss zwischen Studierenden und Betreuenden, denn eine «schnelle und beratende Beantwortung der studentischen Fragen fördert die kontinuierliche, motivierende Auseinandersetzung mit den Lerninhalten und damit den Lernerfolg» (ebd., S. 46). Da Online-Studierende mehrheitlich berufstätig sind, stellt für sie die Integration des Studiums in ihren Lebensalltag zudem eine besondere Herausforderung dar; das Studium muss häufig nicht nur mit dem Beruf, sondern auch mit der Familie in Einklang gebracht werden. Diese Erkenntnis wirkt sich auch auf die Aufgaben der Betreuenden aus: «Die Beratung und Hilfe bei der Organisation ist ein Teil der Betreuung und reicht oftmals bis zu einer Zeit- und Arbeitsplanung mit dem Studierenden» (ebd.). 
Aus den genannten Anforderungen an die Betreuung Online-Studierender leitet Thomaschewski die Forderung nach einem Betreuungskonzept ab,

welches den Studierenden eine schnelle Betreuung und Beratung gewährleistet und eine Sicherheit im Umgang mit den Ansprechpartnern gibt. Alle Belange sollen gebündelt an eine Adresse erfolgen und zeitnah bearbeitet werden. ... Für die konsequente Umsetzung dieser Anforderungen bietet sich ein zweistufiges Betreuungskonzept an. Die Betreuer im First-Level-Support sind wissenschaftliche Mitarbeiter, die den Studierenden als direkte Ansprechpartner zur Verfügung stehen und Fragen weitestmöglich klären. Die fachlich tiefer gehenden Fragen und die Leistungsbewertung übernehmen die Professoren im Second-Level-Support. (ebd.)

In dem als Emder Konzept bezeichneten zweistufigen Betreuungsmodell ermöglicht der First-Level-Support die Nennung fester Ansprechpartner und -zeiten, die Einhaltung garantierter Antwortzeiten (z. B. 24 Stunden für E-Mails und 48 Stunden für Foreneinträge) und die Nutzung unterschiedlicher Kommunikationswege (z. B. Telefon, E-Mail oder Instant Messaging). Die Betreuenden im First-Level-Support, die als Mentoren bzw. Mentorinnen bezeichnet werden, sind für alle technischen und organisatorischen Belange sowie für die Klärung von Lese- oder Verständnisproblemen zuständig. Sie arbeiten im Team selbstständig und weitestgehend eigenverantwortlich. Im Einzelnen obliegen ihnen die folgenden Aufgaben:

- Ansprechpartner für alle Anliegen der Studierenden

- Schnittstellenfunktion zwischen Studierenden und verschiedenen Hochschuleinrichtungen (Immatrikulationsamt, Prüfungsamt etc.)

- Schnittstellenfunktion zwischen Studierenden und Professoren

- Überwachung von Einsendeterminen und Prüfungsvorleistungen

- Vorkorrektur von Einsendeaufgaben

- Sicherstellung der technischen Infrastruktur und technischer Support

- Planung und organisatorische Vorbereitung von Präsenzveranstaltungen und Video-Chats

- Planung der Prüfungszeiträume und der Präsenzstundenpläne

- Studienberatung (ebd., S. 47)

Die im Second-Level-Support betreuenden Professoren/-innen sind für die Durchführung der Online-Lehrveranstaltung verantwortlich. Dies umfasst insbesondere die folgenden, auf die fachlichen Inhalte fokussierten Aufgaben: 
- Erstellung von zusätzlichen Aufgaben und deren Korrekturen

- Durchführung der Präsenzen und der Videochats

- Beantwortung von fachlichen Fragen in den Foren bzw. via E-Mail

- Bewertung der studentischen Leistungen (vgl. ebd.)

Positive Auswirkungen des zweistufigen Konzepts sieht Thomaschewski vor allem im Hinblick auf den für die Betreuung besonders wichtigen Informationsfluss (s. o.). Da die Mitarbeiter/innen im First-Level-Support in ihrer beratenden Funktion nicht für die Bewertung der studentischen Leistungen zuständig sind, wird für die Studierenden "die Schwelle zur Kommunikation herabgesetzt» (ebd., S. 48). Die erhöhte Kommunikationsbereitschaft schlägt sich z. B. in einer regen Nutzung der fachbezogenen Foren nieder, in denen sich die Studierenden untereinander und mit den Betreuenden austauschen. Beobachtet wird auch eine gesteigerte Motivation zur Nutzung nicht-öffentlicher Kommunikationswege (E-Mail und Instant Messaging) zwischen Studierenden und Betreuenden. Der funktionierende Informationsfluss unterstützt die Nachhaltigkeit des Lerneffektes (vgl. ebd.). Gleichzeitig erlernen die Studierenden «die professionelle Nutzung der synchronen und asynchronen Kommunikation, die wiederum eine Voraussetzung für die Aufgabenbearbeitung im räumlich verteilten Team darstellt» (ebd.).

Positive Effekte ergeben sich auch für die betreuenden Professoren/-innen. Aus dem vorgeschalteten First-Level-Support resultiert für sie "ein Betreuungsaufwand, der leicht unter dem des Präsenzstudiengangs liegt» (ebd., S. 47). Insgesamt sieht Thomaschewski im Emder Konzept einen Weg, «eine effiziente und erfolgreiche Betreuung» (ebd., S. 48) im Online-Studium zu gewährleisten.

\section{Split-Tutor-Concept}

Für die Beschreibung der Aufgaben, die das Betreuungspersonal - hier «(Tele-)Tutoren/-innen» genannt - im E-Learning zu erfüllen hat, greifen Prof. Dr. Michael Kerres, Dr. Ilke Nübel und Wanda Grabe auf Modelle aus der Pädagogik zum Lehrverhalten sowie aus der Führungspsychologie zurück (vgl. Kerres/ Nübel/Grabe 2005, S. 339 f.). In Anlehnung an diese Modelle beschreiben die Autoren/-innen zwei Dimensionen tutorieller Aufgaben:

1. fachbezogene Betreuung

○ Klärung von inhaltlichen Fragen, Hilfestellungen bei Verständnisproblemen, Unklarheiten, Missverständnissen

- Hinweise auf Literatur und Hilfsmittel, auf Arbeitstechniken und Methoden

- Hinführung zu Lernaufgaben, Hinweise zur Bearbeitung von Lernaufgaben 
○ Rückmeldung zu Lernaufgaben und zur Vorgehensweise ...

2. personen- bzw. gruppenbezogene Betreuung

- (Unterstützung bei der) Organisation von Lernaktivitäten

- Rückmeldung zum Lernverhalten des Einzelnen/der Gruppe

- Unterstützung bei Konflikten

- Betreuung bei Lernproblemen des Einzelnen/der Gruppe, Studienberatung (ebd., S. 340)

Wie aus der obigen Auflistung hervorgeht, wird die fachbezogene Betreuung eingerichtet, um Verständnisprobleme zu verhindern und den Lernfortschritt zu sichern, während die personen-/gruppenbezogene Betreuung in erster Linie «soziale Präsenz und Kohäsion» (ebd., S. 340) schaffen soll. Je nach Betreuungskonzept können, so die Autoren/-innen, die tutoriellen Tätigkeitsschwerpunkte unterschiedlich angelegt und anhand der beiden Dimensionen eingeordnet werden:

Teilweise werden Tutor/innen vor allem für die fachbezogene Betreuung ... eingesetzt, sie verstehen sich primär als Inhaltsexpert/innen, die bei fachlichen Fragen zu Rate gezogen werden. In anderen Fällen sollen TeleTutor/innen vor allem eine personen- bzw. gruppenbezogene Betreuung übernehmen, sie verstehen sich primär als Coach, der sich für das Wohlbefinden des Einzelnen/der Gruppe verantwortlich fühlt, bei übergreifenden Fragen und Problemen zum Lernprozess einspringt und bei der Erreichung des Lernzieles unterstützt. (ebd., S. 341)

Als «ideal» bewerten die Autoren/-innen das Erreichen hoher Ausprägungen in beiden Dimensionen (s. Abb. 1). Diesem Idealbild steht jedoch ein grundlegendes Dilemma entgegen: Für die personen-/gruppenbezogene Betreuung sind stabile persönliche Beziehungen unerlässlich, die sich häufig erst über einen längeren Zeitraum entwickeln. Wenn allerdings ein/e Tutor/in Studierende über einen langen Zeitraum hinweg betreut, sinkt die Wahrscheinlichkeit, dass diese/r Tutor/in auch für alle inhaltlichen Fragen die fachliche Betreuung leisten kann. Einen Lösungsansatz sehen die Autoren/-innen im Split-Tutor-Concept.

Das Split-Tutor-Concept, das im weiterbildenden Online-Studiengang «Master of Arts in Educational Media» der Universität Duisburg-Essen Anwendung findet, nimmt wegen der geschilderten Schwierigkeiten (s. o.) Abstand von der Vorstellung eines «idealen» Tutors, der die Aufgaben beider Dimensionen erfüllt. Stattdessen ist eine grundsätzliche Trennung von Fach- und Gruppentutoren/-innen vorgesehen (vgl. Kerres/Nübel/Grabe 2005, S. 341; Nübel/Kerres 2004). ${ }^{1}$ 


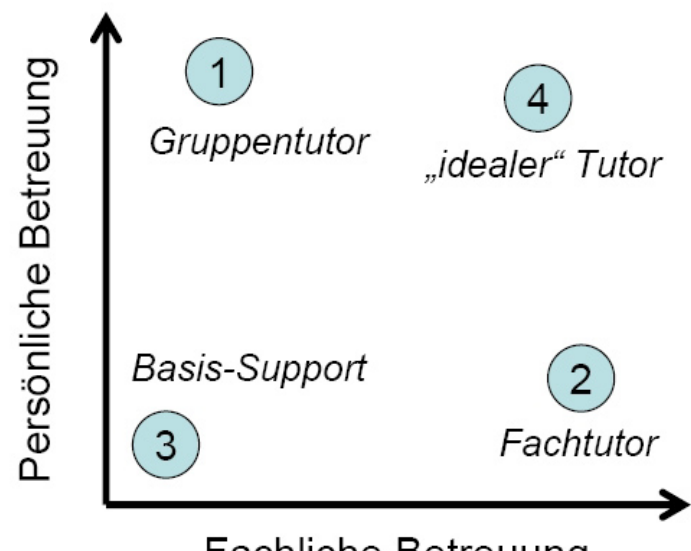

Fachliche Betreuung

Abb. 1: Tutoring-Konzepte (Kerres/Nübel/Grabe 2005, S. 341)

Nach den Erfahrungen der Autoren/-innen haben die Beziehungen der Studierenden untereinander sowie zum/zur Gruppentutor/in «eine wesentliche Bedeutung für einen befriedigenden Studienverlauf» (ebd., S. 347). Den Gruppentutoren/-innen kommt daher bereits während der Auftaktveranstaltung eine wichtige Rolle zu: Sie haben das gegenseitige Kennenlernen innerhalb der Lerngruppen zu fördern und «einen gewissen Grad an Vertrautheit zwischen den Lerngruppenmitgliedern und dem/der Gruppentutor/in herzustellen»(ebd.). Das Fördern von Kommunikation und Austausch bleibt auch im weiteren Verlauf des Studiums eine zentrale Aufgabe der Gruppentutoren/-innen. Dadurch, dass sie den Studierenden von Beginn an vertraut sind, wird deren Hemmschwelle, in Chats und/oder Foren Fragen zu stellen und Probleme zu artikulieren, herabgesetzt.

Fachtutoren/-innen sind für einen Ausschnitt der gesamten Studiengangsinhalte zuständig. Ihre Verantwortlichkeit erstreckt sich dabei nicht nur auf die direkte Betreuung der Studierenden während der Bearbeitungszeit der Lerneinheiten, sondern auch auf die Erstellung der Studienmaterialien vor deren Weitergabe an die Lernenden. Fachtutoren/-innen stehen daher immer auch in engem Kontakt mit den Autoren/-innen der Lehrmaterialien, wodurch die Qualität des Materials gewährleistet wird.

Neben den erwähnten Vorteilen, die das Split-Tutor-Concept für die Studierenden mit sich bringt, sehen Kerres/Nübel/Grabe (2005) als weiteren positiven Effekt eine durch die verteilten Rollen bedingte Entlastung der Betreuenden (vgl. S. 347).

\section{Stufenmodell}

Basierend auf ihren Erfahrungen im kooperativen E-Learning entwickelte Prof. Dr. Gilly Salmon (University of Leicester) ein 5-Stufen-Modell für die Moderation 


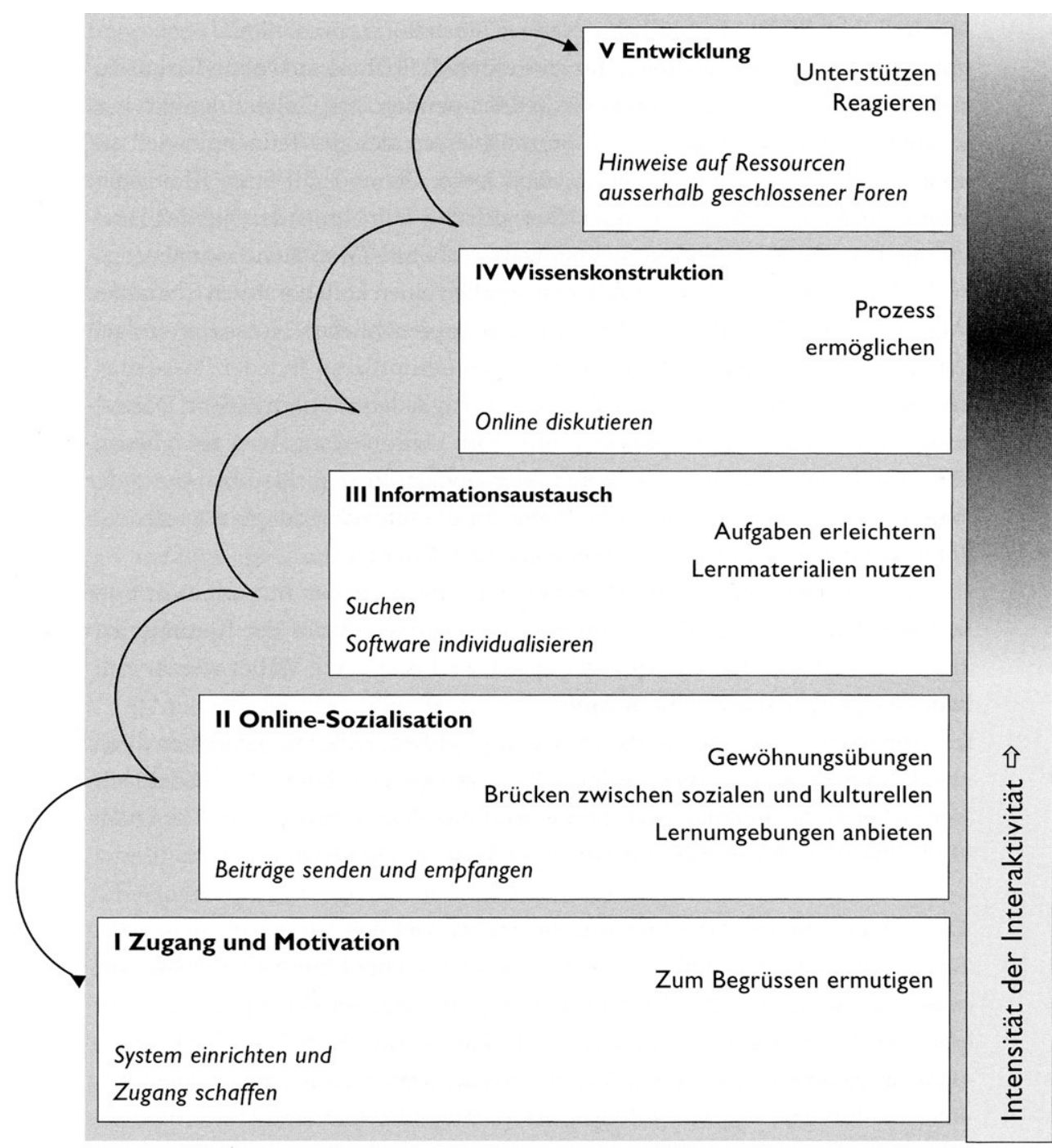

$\begin{array}{ll}\square & \text { E-Moderation } \\ \square & \text { Technischer Support }\end{array}$

Abb. 2: Modell des Online-Lehrens und Lernens in Online-Netzwerken

(Salmon 2004, S. 27)

von Lerngruppen (s. Abb. 2). Sie beschreibt darin sowohl die Entwicklung, die Teilnehmende beim Online-Lernen durchlaufen, als auch die unterschiedlichen Aufgaben, die den sogenannten E-Moderatoren/-innen während dieses Entwicklungsprozesses zukommen und die dazu dienen, «erfolgreiches Lernen zu ermöglichen» (Salmon 2004, S. 26). 
Die fünf Stufen werden von den Mitgliedern einer Lerngruppe nicht zwingend gleichzeitig erreicht bzw. durchlaufen; die Teilnehmenden können auf den einzelnen Stufen unterschiedlich viel Zeit verbringen, und es ist zudem vorstellbar, dass sie mehrmals über die Stufen auf- und absteigen (vgl. Salmon 2004, S. 28).

Nach Salmons Modell entsteht in Online-Lerngruppen bis einschliesslich Stufe III

eine Form von Kooperation, in welcher sich die Teilnehmenden bei der Erreichung ihrer Ziele gegenseitig unterstützen. Auf Stufe IV entstehen kursbezogene Diskussionen, und die Interaktionen erhalten einen kollaborativen Charakter. Auf Stufe $V$ suchen die Teilnehmenden ihren persönlichen Nutzen, verfolgen ihre persönlichen Ziele und reflektieren ihren Lernprozess. ${ }^{2}$ (Salmon 2004, S. 28)

Die Stufen III, IV und V gelten als die für den Lernfortschritt fruchtbarsten und konstruktivsten.

Im Folgenden werden die Aufgaben der E-Moderatoren/-innen, die je nach Stufe variieren, kurz dargestellt.

Auf Stufe I steht die Motivation der Teilnehmenden im Vordergrund. Für die auf den späteren Stufen zentralen interaktiven Prozesse sind eine frühe aktive Beteiligung und die Entwicklung bestimmter Fertigkeiten entscheidend. Dieser Zusammenhang ist den Teilnehmenden zu verdeutlichen. Zudem sollten E-Moderatoren/-innen auf dieser Stufe insbesondere «als Vorbild guter Kommunikation wirken» (Salmon 2004, S. 35). Stufe I gilt als abgeschlossen, wenn möglichst alle Teilnehmenden online arbeiten und den virtuellen Raum häufig besuchen.

Auf Stufe II lernen sich die Teilnehmenden näher kennen und bauen untereinander Beziehungen auf. Dazu «muss ein Vertrauensnetzwerk geknüpft werden, das von physischen Treffen unabhängig ist» (ebd., S. 37). E-Moderatoren/-innen sollen den Teilnehmenden den Wert der Online-Zusammenarbeit vermitteln und ihnen zeigen, wie und was der/die Einzelne zur Gruppenarbeit beitragen kann. Stufe II ist abgeschlossen, «wenn Teilnehmende beginnen, ihre Gedanken online auszutauschen. Damit ist die Basis für den zukünftigen Informationsaustausch und die Wissenskonstruktion gelegt» (ebd., S. 40).

Im Mittelpunkt von Stufe III stehen die Aktivitäten der Teilnehmenden. E-Moderatoren/-innen sollten ihnen hier Arbeitsaufträge erteilen, die die «Interaktion mit dem Kursinhalt, mit den E-Moderatoren sowie mit anderen Kursteilnehmenden» (ebd.) erfordern. Bevor jedoch interagiert und kooperiert wird, benötigen die Teilnehmenden Kenntnisse der zielgerichteten Informationssuche. Die E-Moderatoren/-innen machen sie daher mit entsprechenden Strategien und Werkzeugen vertraut. Stufe III ist beendet, «wenn Teilnehmende gelernt haben, 
Informationen zu finden ... und wenn die Anzahl derjenigen minimal ist, welche passiv lesen («lurkers»), durchblättern und nur indirekt lernen» (ebd., S. 44).

Prozesse der Wissenskonstruktion sind das primäre Ziel auf Stufe IV. Die Teilnehmenden sollen über verschiedene Perspektiven und Beispiele eigene interne Wissensmuster entwickeln, diese mit persönlichen Erfahrungen verknüpfen und ein vertieftes Verständnis erlangen. Diese Phase erfordert eine besonders feinfühlige Unterstützung durch die E-Moderatoren/-innen, die das richtige Mass an Struktur vorgeben müssen:

Gute E-Moderatorinnen fassen von Zeit zu Zeit zusammen, geben einen Überblick über die Standpunkte und schlagen neue Themen vor, wenn sich Diskussionen verlieren. Sie entwickeln neue Gedankenfäden, führen neue Schwerpunkte ein und schlagen alternative Vorgehensweisen vor. (Salmon 2004, S. 46)

Stufe IV kann als abgeschlossen betrachtet werden, wenn eine gemeinsam erarbeitete Online-Aktivität «zu einem vereinbarten Ergebnis geführt hat» (ebd., S. 48).

Auf Stufe $\vee$ geht es schliesslich darum, «Einsichten herbeizuführen sowie Erkenntnisse und aufgebautes Wissen kritisch zu überdenken und zu beurteilen» (ebd., S. 129). Durch die Reflexion soll die Nachhaltigkeit des (individuellen und gruppenbezogenen) Online-Lernens gefördert werden. Die Teilnehmenden übernehmen auf dieser Stufe selbst die Verantwortung für das eigene Lernen und das ihrer Gruppe, und "Teilnehmende mit mehr Erfahrung werden oft zu hilfreichen Ratgeberinnen für neu Ankommende» (ebd., S. 48).

Unabhängig von der Stufe, auf der sich die Teilnehmenden befinden, sollten E-Moderatoren/-innen laufend Rückmeldungen zu Lernfortschritten geben, Verbesserungsvorschläge einbringen und «sicherstellen, dass der sozial orientierte Gesprächsaspekt für jene, die es wünschen, während des ganzen Kurses beibehalten wird» (ebd., S. 40). Letzteres kann z. B. über die Einrichtung eines «Bar»- oder "Café»-Forums gewährleistet werden. Permanente Beachtung erfordert auch das Tempo, in dem die Teilnehmenden durch die Stufen gehen; es darf weder zu hoch noch zu niedrig sein. Ziel all jener Aktivitäten ist es, «die Lerngruppen über die fünf Phasen hinweg selbstständig und unabhängig von aussengeleiteter Moderation zu machen, indem die Vernetzung der Guppenmitglieder und die Eigenständigkeit der Gruppe gezielt aufgebaut wird» (Schenk 2004, S. 219).

\section{Teaching-Presence-Modell}

Zur Erläuterung des Teaching-Presence-Gedankens ist zunächst eine Beschreibung des zugrunde liegenden Konzepts der Community of Inquiry erforderlich. 
Die kanadischen Wissenschaftler Prof. Dr. Randy Garrison (University of Calgary) und Prof. Dr. Terry Anderson (Athabasca University) beschreiben eine Community of Inquiry als eine Gemeinschaft, in der

students listen to one another with respect, build on one another's ideas, challenge one another to supply reasons for otherwise unsupported opinions, assist each other in drawing inferences from what has been said, and seek to identify one another's assumptions. A community of inquiry attempts to follow the inquiry where it leads rather than being penned in by the boundary lines of existing disciplines. (Garrison/Anderson 2003, S. 27, nach Lipman 1991)

Das Community-of-Inquiry-Konzept ist als eine Ausprägung des Learning-Community-Ansatzes zu betrachten. Beide Konzepte stimmen in grundlegenden Ziele und wesentlichen Merkmalen überein. So finden sich in den Communities, die sowohl in Präsenzform als auch virtuell realisiert werden können, Menschen zusammen, die sich gemeinsam mit einem bestimmten Thema auseinandersetzen wollen. Angestrebt wird die Einbeziehung und umfassende Gleichberechtigung aller Beteiligten; durch aktives und interaktives Lernen soll ein tieferes Verständnis der Inhalte erreicht, das kollektive Wissen vermehrt, die individuelle Wissensentwicklung gefördert sowie Selbststeuerungskompetenz und Eigenverantwortung entwickelt werden. Lernen im sozialen Austausch ist demnach ein zentrales Merkmal beider Community-Ansätze.

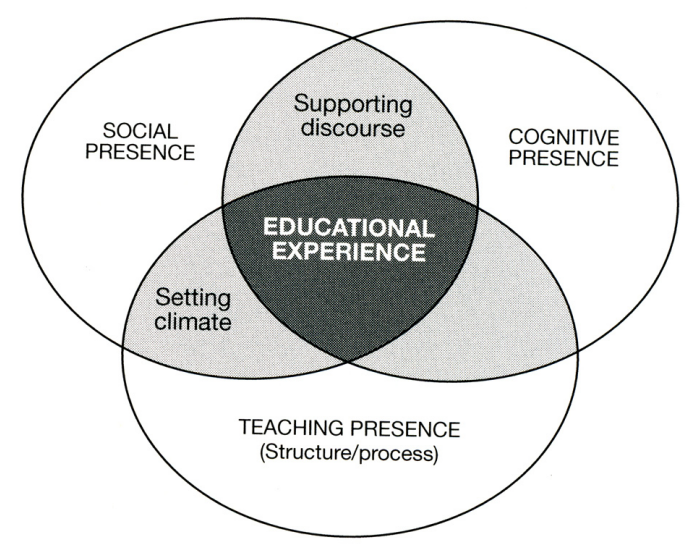

Abb. 3: Strukturelle Hauptelemente in Communities of Inquiry (Garrison/Anderson 2003, S. 28)

Das Community-of-Inquiry-Konzept basiert auf dem Zusammenspiel dreier struktureller Hauptelemente, die von Garrison/Anderson (2003) als Cognitive Presence, Social Presence und Teaching Presence bezeichnet werden (s. Abb. 
3) und die sich förderlich auf die Beteiligten bzw. auf den Lehr-Lern-Prozess auswirken können.

Cognitive Presence wird als «the extent to which the participants in any particular configuration of a community of inquiry are able to construct meaning through sustained communication» (Garrison/Anderson/Archer 2000, S. 94) definiert. Sie gilt als die für den Lernerfolg wesentlichste Komponente und als Voraussetzung für die Fähigkeit des kritischen Denkens, eines der grundlegenden Ziele z. B. in der universitären Bildung. Da Kommunikation eine zentrale Rolle im Entstehen von Cognitive Presence spielt, ist in einer Online-Community of Inquiry neben den diesbezüglichen medialen Gegebenheiten das sozial-emotionale Umfeld von grosser Bedeutung. Dieser soziale Rahmen wird bestimmt durch Social Presence, das zweite Kernelement in Communities of Inquiry (vgl. ebd.).

Als Social Presence beschreiben Garrison/Anderson/Archer (2000) «the ability of participants in a community of inquiry to project themselves socially and emotionally, as «reals people (i.e., their full personality), through the medium of communication being used» (S. 94). Social Presence dient in erster Linie der Unterstützung des innerhalb der Community geführten Diskurses und dadurch der Förderung von Cognitive Presence. Eine besondere Herausforderung stellen dabei die Besonderheiten der computervermittelten, textbasierten Kommunikation (z. B. das Fehlen nonverbaler Signale) dar.

Die Ausrichtung der beiden beschriebenen Komponenten auf den angestrebten Lehr-Lern-Prozess wird schliesslich durch das dritte konstituierende Element einer Community of Inquiry, Teaching Presence, sichergestellt, die als «design, facilitation, and direction of cognitive and social processes for the purpose of realizing personally meaningful and educationally worthwhile learning outcomes» (Anderson u. a. 2001, S. 5) verstanden wird. Zwar finden auch ohne eine solche aktive Unterstützung Lernprozesse statt, doch nur mit ihr können die vorhandenen Potenziale komplexer kooperativer E-Learning-Szenarien optimal ausgeschöpft werden (vgl. ebd.).

Im Rahmen Teaching Presence sind Lehrende in Communities of Inquiry in drei Aufgabenfeldern tätig: Design and Organization, Facilitating Discourse und Direct Instruction.

Der Bereich Design and Organization betrifft die Konzeption und Gestaltung von Lehr-Lern-Szenarien, wobei unter dem Begriff Design die im Vorfeld eines E-Learning-Angebots anfallenden Entscheidungen und Tätigkeiten subsumiert werden, während sich der Begriff Organization auf die im Verlauf des Lehr-LernProzesses notwendigen Anpassungen bezieht. In der Organization-Phase haben die Lehrenden durch kontinuierliches Anpassen der Rahmenbedingungen sicherzustellen, dass die Lernenden über «an appropriate degree of control over the management and monitoring of their activities and learning» (Garrison/Anderson 2003, S. 78) verfügen. Die Voraussetzungen dafür werden in der Design- 
Phase geschaffen, die vom angestrebten Ideal der Übernahme von Teaching Presence durch Lernende geleitet werden: «Responsibility and control must naturally evolve as the learner progresses socially and cognitively» (ebd., S. 78). Unter Facilitating Discourse werden alle Aktivitäten verstanden, die der Entwicklung des Diskurses und damit der Aufrechterhaltung des Interesses, der Motivation und der Beteiligung der Lernenden dienen. Die Unterstützung des Diskurses ist entscheidend für die Lerngemeinschaft sowohl auf der Gruppenebene als auch auf individueller Ebene, weshalb Garrison/Anderson (2003) diesen Bereich auch als "heart of the e-learning experience» (S. 68) bezeichnen. Den Diskurs angemessen zu unterstützen erfordert Sensibilität seitens der Lehrenden, da sich zu viel oder zu wenig Teaching Presence in diesem Zusammenhang nachteilig auswirken kann. Facilitating Discourse beinhaltet neben sozialen Aspekten auch fachliche Hilfestellungen, weil sich im Diskurs nicht nur personen-/ gruppenbezogene, sondern auch inhaltliche Aspekte manifestieren. Facilitating Discourse erfordert somit neben dem Herstellen und Aufrechterhalten eines offenen, konstruktiven Lernklimas auch das Beobachten und Unterstützen des (einander) Verstehens. Eine strikte Trennung der personen-/gruppenbezogenen Betreuung von der fachbezogenen Betreuung, wie sie in anderen Konzepten verfolgt wird, ist in Communities of Inquiry dadurch nicht vorstellbar.

Der Bereich Direct Instruction betrifft in erster Linie die inhaltliche Unterstützung der Lernenden. Garrison/Anderson (2003) unterstreichen die Wichtigkeit eines erfahrenen, fachlich kompetenten Lehrenden, der Ideen und Gedankengänge beurteilen, Lernaktivitäten strukturieren, Missverständnisse aufklären und Hinweise auf zusätzliche Informationsquellen geben kann und so zur Wissenskonstruktion und Sinnzuweisung beitragen kann. Sie grenzen sich damit von Konzepten $a b$, in denen Lehrende in virtuellen Lehr-Lern-Szenarien ausschliesslich als beratende und moderierende Lernbegleiter/innen gesehen werden, die nicht für Wissensvermittlung und instruktionale Anleitung zuständig sind. Angestrebt wird stattdessen eine flexiblere Herangehensweise, die ein situatives Betreuen ermöglicht: "At times the teacher may be a guide on the side ... at times a sage on the stage ... - or, at other times, something in between in the role of an active moderator» (ebd., S. 81).

Garrison/Anderson (2003) betonen, dass in einer Community of Inquiry nicht nur Lehrende dazu in der Lage sind, Teaching Presence auszuüben, sondern jede/r Beteiligte die Verantwortung für den Fortschritt der Gruppe trägt und zur Teaching Presence beitragen kann und soll (die deshalb auch ausdrücklich nicht als Teacher Presence bezeichnet wird). Diese Möglichkeit ist nicht nur für die Förderung der Gruppe, sondern insbesondere auch für die individuelle Entwicklung elementar, da jede/r Einzelne durch Teaching-Presence-Aktivitäten die Fähigkeit zum selbstgesteuerten Lernen steigert. Die im Zeitverlauf zunehmende Übernahme von Teaching Presence durch Lernende in Communities of In- 
quiry (s. Abb. 4) wird durch eine Reihe empirischer Untersuchungen belegt (vgl. Anderson u. a. 2001; Heckman/Annabi 2003; Rourke/Anderson 2002). Mit der Verlagerung der Teaching Presence von den Lehrenden zu den Lernenden sind bestimmte Anforderungen an alle Beteiligten verbunden: Lernende müssen Verantwortung für den Lernprozess übernehmen und Lehrende müssen dazu bereit sein, dies auch zuzulassen - «Lehrende zeigen also Verantwortung, indem sie Verantwortung abgeben» (Czerwionka/de Witt 2006, S. 128).

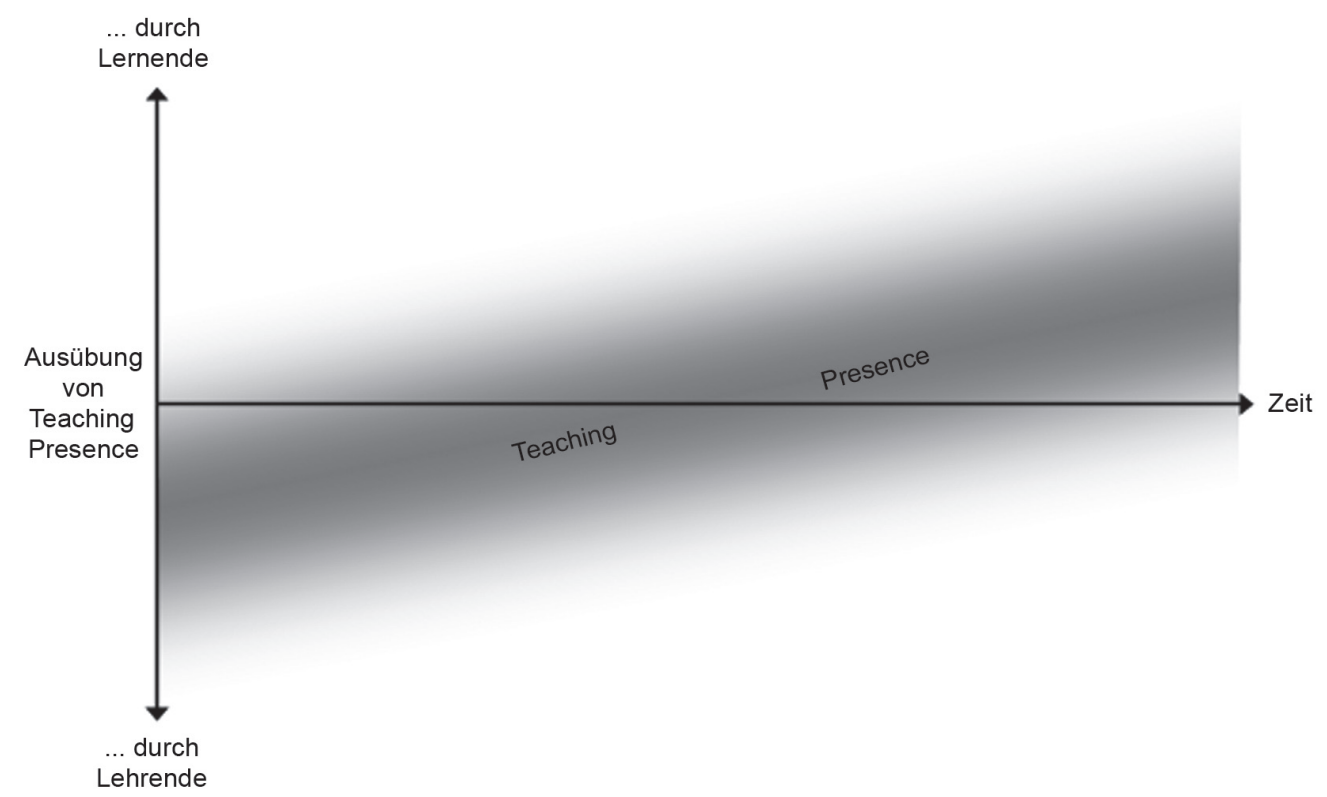

Abb. 4: Ausübung von Teaching Presence im Zeitverlauf (Czerwionka/de Witt 2006, S. 129)

Das Ausüben von Teaching Presence durch Lernende macht die Betreuung durch Lehrende keineswegs überflüssig; ihnen obliegen kontinuierlich die Strukturierung, Gestaltung und Beurteilung der Lernerfahrungen. Pädagogischdidaktische Vorteile des Teaching-Presence-Modells bestehen u.a. darin, dass die Lernenden durch die allmähliche Übernahme von Betreuungsleistungen Kompetenzen erwerben können hinsichtlich der Organisation von kooperativen E-Learning-Szenarien, der Initiierung und Durchführung von Diskursen sowie der Wissenskonstruktion und -anwendung. "Situatives Betreuen wird so zum zentralen Merkmal im Lernprozess einer Online-Community of Inquiry» (Czerwionka/de Witt 2006, S. 129). 


\section{Zusammenfassung und Vergleich}

Wie stark sich die vier vorgestellten Betreuungskonzepte voneinander unterscheiden, wird besonders anhand der Aufgaben deutlich, die dem/der/den Betreuenden jeweils zukommen.

Allgemein können vier während der Durchführung virtueller Bildungsangebote bedeutsame grundsätzliche Rollen und entsprechende Aufgabenfelder für Betreuende unterschieden werden (vgl. Arnold u. a. 2004; Czerwionka/de Witt 2006; Kerres/Nübel/Grabe 2005):

- Fachbezogene Betreuung

Aufgabenfeld: Anregung und Unterstützung der fachlichen Auseinandersetzung mit den Lerninhalten (z. B. Klärung inhaltlicher Fragen, Hilfestellung bei Verständnisproblemen)

- Personen-/Gruppenbezogene Betreuung

Aufgabenfeld: Unterstützung selbstgesteuerten Lernens (z. B. bei der Organisation von Lernaktivitäten, bei der Auswahl passender Lernwege und -strategien, im Zeitmanagement, bei Motivationsproblemen) und Begleitung des Gruppenprozesses (z. B. Herstellen sozialer Bezüge zu Lernenden und Lehrenden, Hilfestellung bei Lernproblemen und/oder Konflikten in der Gruppe)

- Organisatorische Betreuung

Aufgabenfeld: Gewährleistung förderlicher Rahmenbedingungen (z. B. Bereitstellung von Lernmaterialien, Administration der Lernumgebung)

- Technische Betreuung

Aufgabenfeld: Hilfestellung bei technischen Problemen

Dass den Betreuenden in den verschiedenen Konzepten unterschiedliche Rollen und Aufgaben zukommen, lässt sich z. T. bereits an den für sie verwendeten Bezeichnungen ablesen. So nennt beispielsweise Thomaschewski die Mitarbeiter/innen im (weniger auf die fachbezogene Betreuung ausgerichteten) FirstLevel-Support «Mentoren», während Salmon (2004) ausschliesslich von «Moderatoren» bzw. «Moderatorinnen» spricht und Garrison/Anderson (2003) sowohl die Begriffe «teacher» als auch «active moderator» gebrauchen.

Die Eintragungen unter «Betreuungsqualität und -quantität» geben an, ob das jeweilige Betreuungskonzept explizit eine von der Entwicklung der Lernenden abhängige Anpassung der Betreuungsleistungen vorsieht. Im Emder Konzept und im Split-Tutor-Concept wird eine solche Anpassung nicht ausdrücklich erwähnt; die Aufgaben der Betreuenden werden - auch im Falle einer längerfristigen Betreuung, wie sie z. B. dem/der Gruppentutor/in im Split-Tutor-Concept zukommt - als gleichbleibend beschrieben. Diese Konzepte werden deshalb als 
«statisch» bezeichnet. Im Gegensatz dazu stellt in den «dynamischen» Konzepten die Entwicklung der Lernenden ein zentrales Element dar: Sowohl im Stufenmodell als auch im Teaching-Presence-Modell wird angestrebt, die Selbststeuerungsfähigkeit der Lernenden schrittweise zu fördern und analog dazu die verschiedenen Formen äusserer Anleitung und Unterstützung allmählich auszublenden. Diese Einstellung entspricht einer heute beim Einsatz kooperativer netzbasierter Lernmethoden häufig formulierten Forderung (vgl. ReinmannRothmeier/Mandl 2002, S. 52 f.; Euler 2001, S. 16).

Tabelle 1 verdeutlicht, welche der oben genannten Rollen und Aufgabenfelder in den vorgestellten Konzepten jeweils im Vordergrund stehen. ${ }^{3}$

BETREUUNGSKONZEPTE

\begin{tabular}{|l|c|l|l|c|}
\hline $\begin{array}{l}\text { Rollen und Betreuungsqualität/- } \\
\text { quantität }\end{array}$ & $\begin{array}{l}\text { Emder } \\
\text { Konzept }\end{array}$ & $\begin{array}{l}\text { Split- } \\
\text { Tutor- } \\
\text { Konzept }\end{array}$ & $\begin{array}{l}\text { Stufen- } \\
\text { modell }\end{array}$ & $\begin{array}{l}\text { Teaching- } \\
\text { Presence- } \\
\text { Modell }\end{array}$ \\
\hline Fachbezogene Betreuung & $\bullet_{A}$ & $\bullet_{A}$ & & $\bullet$ \\
\hline Personen-/Gruppenbezogene Betreuung & $\bullet_{B}$ & $\bullet_{B}$ & $\bullet$ & $\bullet$ \\
\hline Organisatorische Betreuung & $\bullet_{B}$ & & & \\
\hline Technische Betreuung & $\bullet_{B}$ & & & \\
\hline Betreuungsqualität und -quantiät & statisch & statisch & dynamisch & dynamisch \\
\hline
\end{tabular}

Tab. 1: Rollen und Betreuungsqualität/-quantität in verschiedenen Betreuungskonzepten

\section{Perspektiven für die mentorielle Betreuung im Fernstudium}

Im Folgenden werden sowohl auf der Grundlage der geschilderten Konzepte als auch unter Bezugnahme auf verschiedene Studien zum computergestützten und/oder kollaborativen Lernen Empfehlungen für zukünftige Betreuungsszenarien formuliert. Im Mittelpunkt stehen dabei drei zentrale Aspekte mentorieller Betreuung: die Gestaltung von Mentoriaten, die Gestaltung der Zusammenarbeit der Betreuenden und die zu berücksichtigenden Anforderungen an Mentoren/-innen.

\section{Gestaltung von Mentoriaten}

Wie aktuelle Erfahrungen an der FernUniversität in Hagen zeigen, stösst ein Betreuungsmodell, dem die Konzepte des Blended Learning und des kooperativen Lernens zugrunde liegen, sowohl unter den Studierenden als auch in den Reihen der Betreuenden auf breite Akzeptanz (vgl. de Witt/Czerwionka/Mengel 
2006). Im Blended Learning vereinen sich die (im System der FernUniversität seit mehr als 30 Jahren gewachsenen und bewährten) Formen der Präsenzbetreuung und neue, virtuelle Betreuungsangebote. Ziel eines ganzheitlichen Betreuungskonzepts muss es sein, die spezifischen Vorzüge beider Betreuungsformen optimal in Einklang zu bringen.

Präsenzveranstaltungen ermöglichen den direkten persönlichen Kontakt der Studierenden zu ihren Kommilitonen/-innen und zu Betreuenden. Die Nachfrage nach dieser Betreuungsform scheint insbesondere in der Anfangsphase des Fernstudiums hoch zu sein (vgl. de Witt/Czerwionka/Mengel 2006). Erklären lässt sich dieser Bedarf durch eventuelle anfängliche Unsicherheiten sowohl in Bezug auf die neue, ungewohnte Studiensituation als auch hinsichtlich des Umgangs mit dem Studienmaterial. Das Angebot an Präsenzbetreuung sollte daher zu Beginn des Studiums besonders ausgeprägt sein. Dabei muss - neben der notwendigen inhaltlichen Unterstützung - Wert darauf gelegt werden, den Studierenden die für das erfolgreiche Absolvieren ihres Fernstudiums erforderlichen Kompetenzen zu vermitteln. Zu diesen Kompetenzen zählen nicht nur übergreifende «Schlüsselkompetenzen» wie die

- Kompetenz zu wissenschaftlichem Arbeiten oder die

- Kompetenz zu selbstgesteuertem Lernen,

sondern auch solche, die sich in der Auseinandersetzung mit den Studiengangsinhalten entwickeln, wie

- Reflexionskompetenz,

- Methodenkompetenz und

- Handlungskompetenz.

Die Vermittlung der genannten Schlüsselkompetenzen ist an keine speziellen Inhalte gebunden und muss daher nicht zwingend von Fachmentoren/-innen oder Kurs-/Modulbetreuern/-innen vorgenommen werden. Zahlreiche Studienzentren verfügen bereits über Erfahrungen in der Organisation und Durchführung entsprechender Veranstaltungen. Schlüsselkompetenzschulungen könnten das Betätigungsfeld der Mitarbeiter/innen der allgemeinen Studienberatung (Studienberater/innen) erweitern.

Mit fortschreitender Studiendauer kann der Anteil virtueller Elemente am gesamten Betreuungsangebot gesteigert werden. Voraussetzung dafür ist die diesbezügliche hohe Aufgeschlossenheit aller Beteiligten. Für die Betreuenden sind virtuelle Angebote aufgrund ihrer hohen Effizienz attraktiv: Von der Verbreitung von Studienmaterialien über das Internet über den Dialog per E-Mail bis hin zur Diskussion in Chats und Foren bieten ihnen Neue Medien vielfältige 
Möglichkeiten der Begleitung und Steuerung des Lernprozesses. Doch auch Studierende können von der virtuellen Betreuung profitieren - insbesondere dann, wenn in ihr das zweite oben genannte Basiskonzept, das kooperative Lernen, umgesetzt wird: Durch Kommunikation und Kooperation kann nicht nur die inhaltliche Arbeit unterstützt, sondern auch der Anonymität (der «Entpersonalisierung») der Beteiligten entgegengewirkt werden. Höhere Lernerfolge, verbesserte soziale Kompetenzen und eine gesteigerte Lernmotivation sind die Folge (vgl. Euler 2001; Johnson/Johnson/Stanne 2000). Je mehr die bereits von Otto Peters geforderten «Gelegenheiten für soziales Lernen» (Peters 1976, S. 141) geschaffen werden, desto stärker kann sich Partizipation vollziehen.

Trotz unterschiedlicher Vorzüge stellen Präsenzbetreuung und virtuelle Betreuung immer weniger ein Gegensatzpaar dar. "Live Collaboration Tools» wie z. B. virtuelle Klassenzimmer erlauben ein audio- und videobasiertes synchrones Zusammentreffen und -arbeiten mehrerer Personen im virtuellen Raum. Die Übertragung von Bild und Ton fördert die soziale Präsenz der Beteiligten in der Lernumgebung und gleicht dadurch einen Nachteil rein textbasierter Angebote wie Diskussionsforen und Chats aus. Anders als z. B. Foren eignen sich Live Collaboration Tools jedoch wie «reale» Präsenzveranstaltungen eher für geringe Gruppengrössen, was angesichts der teilweise hohen Studierendenzahlen in einem Kurs/Modul die Frage nach einer sinnvollen Integration derartiger Tools in das Betreuungskonzept eines Fernstudiums aufwirft. Denkbar ist beispielsweise ihr Einsatz im Rahmen der Gruppenpuzzlemethode, in der Stamm- und Expertengruppen in unterschiedlicher Besetzung, aber mit überschaubaren Grössen gebildet werden, zusammenkommen und sich austauschen (s. http://www.e-teaching.org/didaktik/konzeption/methoden/gruppenlernen/puzzle/).

Auch für die Übertragbarkeit anderer Betreuungskonzepte auf den jeweiligen organisationalen Kontext spielt die Gruppengrösse eine wichtige Rolle. Je umfangreicher und anspruchsvoller die Aufgaben der Betreuenden sind, desto kleiner müssen die Gruppen sein, damit diese Aufgaben erfüllbar bleiben. Gleichzeitig gilt, dass Kommunikation und Zusammenarbeit im kooperativen Lernen immer auch an inhaltliche Aspekte gebunden sind, weshalb Garrison/Anderson (2003) den Diskurs in den Mittelpunkt ihrer Überlegungen zur Betreuung im ELearning stellen. Eine solche diskursbezogene Betreuung erfordert neben dem Herstellen und Aufrechterhalten eines offenen, konstruktiven Lernklimas auch das Beobachten und Unterstützen des (einander) Verstehens. Die Trennung der personen-/gruppenbezogenen Betreuung von der fachbezogenen Betreuung, wie sie in den übrigen vorgestellten Konzepten verfolgt wird, ist im Betreuungskonzept von Garrison/Anderson (2003) nicht vorstellbar. Mit seiner intensiven, drei der vier unterschiedlichen Rollen umfassenden Betreuungsqualität dürfte zwar auch ihr Konzept bei grossen Gruppen an seine Grenzen stossen, doch der Diskursbezug sowie das Ziel, die Verantwortung für den Lernprozess suk- 
zessive von den Lehrenden/Betreuenden an die Lernenden zu übertragen, sind Elemente, die generell im Fernstudium berücksichtigt werden sollten. Gerade für den letztgenannten Aspekt ist die Vermittlung grundlegender Kompetenzen (s. o.) unabdingbar.

Zusammengefasst können die folgenden Empfehlungen für die Gestaltung von Mentoriaten gegeben werden:

- Basiskonzepte:

- Blended Learning

- Kooperatives Lernen

- Diskursbezogene Betreuung

- Präsenzbetreuung:

- Insbesondere zu Studienbeginn

- Ziele: Inhaltliche Unterstützung und Unterstützung des Lernprozesses durch Vermittlung notwendiger Kompetenzen (Reflexions-, Methodenund Handlungskompetenz durch Fachmentoren/-innen und/oder Kurs-/ Modulbetreuer/innen, Schlüsselkompetenzen z. B. durch Studienberater/ innen)

- Virtuelle Betreuung:

- Insbesondere im fortgeschrittenem Studium

- Ziele: Inhaltliche Unterstützung und Unterstützung des Lernprozesses durch Übertragen der Verantwortung für den Lernprozess von den Betreuenden auf die Studierenden

- Grossgruppenbetreuung über Lernumgebungen wie z. B. moodle

- Kleingruppenbetreuung über Live Collaboration Tools wie z. B. virtuelle Klassenzimmer

- Individualbetreuung per E-Mail

\section{Gestaltung der Zusammenarbeit der Betreuenden}

Im Rahmen einer nach den obigen Empfehlungen gestalteten diskursbezogenen Betreuung sollen Kommunikations- und Kooperationsprozesse zwischen Studierenden und ihren Kommilitonen/-innen sowie zwischen Studierenden und ihren Betreuenden angeregt und unterstützt werden. Trotz der (logischen) Konzentration auf die/den Studierende/ $\mathrm{n}$ darf in den Überlegungen zum zukünftigen Betreuungskonzept die Beziehung der Betreuenden untereinander nicht vernachlässigt werden. Die Integration entsprechender Angebote in ein umfassendes Betreuungskonzept schafft die Grundlage für einen kontinuierlichen Informationsfluss bezüglich studien-/betreuungsrelevanter Aspekte. Be- 
treuungsprozesse können optimiert und darüber hinaus persönliche Kontakte gefördert werden.

Denkbar sind verschiedene Möglichkeiten der Förderung von Kommunikation und Kooperation, wie z. B.

- Bereitstellung eines webbasierten Diskussionsforums mit integriertem ChatTool

- Präsenztreffen in Form von Informations- bzw. Mentoren/-innenworkshops

- Regelmässige Koordinierungstreffen sowohl in Präsenzform als auch in Form von Online-Meetings

Ein derartiges Angebot allein führt jedoch nicht zwingend zu mehr Kooperation (vgl. de Witt/Czerwionka/Mengel 2006). Sinnvoll scheint daher die Einrichtung einer koordinierenden Instanz in Form einer/eines modulübergreifend tätigen Mitarbeiterin/Mitarbeiters für die Betreuungsorganisation (Betreuungskoordinator/in) als Schnittstelle zwischen Mentoren/-innen und Kurs-/Modulbetreuern/-innen. Der Verantwortungsbereich eines/einer solchen Betreuungskoordinators/-koordinatorin sollte die folgenden Aufgaben umfassen:

- Kommunikation/Austausch/Informationsfluss zwischen Mentoren/-innen und Kurs-/Modulbetreuern/-innen fördern (z. B. durch Administration eines virtuellen Mentoren/-innenforums und Organisation regelmässiger Meetings)

- Betreuungsstandards abstimmen und anpassen (z. B. feste Erreichbarkeit, feste Ansprechpartner/innen und Zuständigkeiten, verbindliche Antwortzeiten)

- Präsenzveranstaltungen koordinieren

- Auftaktveranstaltungen organisieren

- Weiterbildungsangebote für Betreuende organisieren/ermöglichen

- Studiengang auf Messen/Kongressen repräsentieren

- Studiengangsberatung durchführen

\section{Anforderungen an Mentoren/-innen}

Vor dem Hintergrund des hier empfohlenen diskursbezogenen, kommunikations- und kooperationsbasierten Betreuungskonzepts gewinnen Medienkompetenz sowie sozial-kommunikative Kompetenz an Bedeutung.

Die verstärkte Berücksichtigung virtueller Betreuungsangebote und der Einsatz neuer, veränderter Angebotsformen wie z. B. Live Collaboration Tools (s. o.) erfordert die umfassende Medienkompetenz der Betreuenden, die in virtuellen Szenarien unterschiedliche Rollen einnehmen: 
Sie sind selbst Mediennutzer, darüber hinaus jedoch gestalten sie durch die Auswahl von Inhalten und Werkzeugen die Lernsituation. Schliesslich müssen sie nicht nur selbst kompetent mit Medien umgehen können, sondern diese Kompetenz auch bei den Lernenden fördern. (Arnold u. a. 2004, S. 154)

Aufgrund ihrer gewachsenen Bedeutung darf die verlangte Medienkompetenz nicht - wie häufig zu beobachten - nur unscharf, oberflächlich oder gar nicht definiert werden. Stattdessen sind sämtliche Dimensionen von Medienkompetenz zu beachten. Nach Baacke (1999) lassen sich vier wesentliche Aspekte unterscheiden:

- Medienkunde,

- Medienkritik,

- Mediennutzung und

- Mediengestaltung (vgl. Baacke 1999, S. 31 ff.).

Medienkompetenz umfasst somit technische und pädagogische Aspekte. Ausser Bedienungskenntnissen sind vor allem kritisch-analytische und gestalterische Fähigkeiten gefordert: Medial vermittelte Inhalte müssen verstanden und bewertet und Medien den eigenen Interessen entsprechend genutzt werden können (vgl. Arnold u. a. 2004, S. 154 f.; Rautenstrauch 2001, S. 82; Schröder/ Wankelmann 2002, S. 22).

Zur bestmöglichen Nutzung der Potenziale virtueller Kommunikationsformen ist zusätzlich zur Medienkompetenz eine ausgeprägte sozial-kommunikative Kompetenz notwendig. Betreuende müssen «nicht nur die technische, sondern auch die sozialpsychologische Seite der Kommunikation beherrschen» (Schröder/ Wankelmann 2002, S. 26). Zu den Besonderheiten und speziellen didaktischen Implikationen der virtuellen Kommunikation gehören beispielsweise

- die Vor- und Nachteile der unterschiedlichen Kommunikationsarten,

- mögliche Kommunikationsprobleme ...,

- Methoden, um das Gruppengefühl von virtuellen Arbeitsgruppen zu unterstützen, sowie

- die Metabotschaften und die «Netiquette〉 der einzelnen Werkzeuge. (Arnold u. a. 2004, S. 156; vgl. Rautenstrauch 2001, S. 83)

Betreuende müssen diese Besonderheiten kennen und sie situationsabhängig angemessen berücksichtigen - sowohl in der Kommunikation mit Studierenden als auch in der mit anderen Betreuenden. 
Ebenfalls zum sozial-kommunikativen Bereich ist die Fähigkeit der Betreuenden zu zählen, sich im Sinne einer dynamischen Betreuung entsprechend den im Verlauf des Lernprozesses wachsenden Kompetenzen der Studierenden mehr und mehr zurückzuziehen:

Lernende sind aufgefordert, Verantwortung für den Lernprozess zu übernehmen, und Lehrende müssen dazu bereit sein, dies auch zuzulassen - Lehrende zeigen also Verantwortung, indem sie Verantwortung abgeben. (Czerwionka/de Witt 2006, S. 129)

Die Vielzahl und Komplexität der hier beschriebenen Kompetenzen verdeutlicht letztlich die Notwendigkeit entsprechender Schulungen für Betreuende. Zwar existieren schon seit Längerem Qualifizierungsangebote, doch diese

sind sehr heterogen und von sehr unterschiedlicher Qualität, nicht nur hinsichtlich der zeitlichen Dauer, sondern auch in Bezug auf ihre didaktischen und inhaltlichen Schwerpunkte. Verbindliche (Qualitäts-)Standards oder allgemein anerkannte Zertifizierungen gibt es noch nicht, entsprechende Entwicklungen werden zumindest noch einige Zeit in Anspruch nehmen (Arnold u. a. 2004, S. 167)

Bedarfsanalysen und die anschliessende Auswahl passender Schulungsangebote stellen daher eine der Hauptaufgaben von Betreuungskoordinatoren/-innen dar (s. o.).

\section{Fussnoten}

1 Je nach individuellen Interessen und Kenntnissen können Tutoren/-innen jedoch auch im Split-Tutor-Concept sowohl die Rolle von Gruppentutoren/-innen als auch die von Fachtutoren/-innen für bestimmte Fragen übernehmen, wodurch eine "Matrixorganisation der Betreuung» (Kerres/Nübel/Grabe 2004, S. 347) entsteht.

2 Der Balken am rechten Rand der Abbildung gibt den Grad der zu erwartenden Interaktivität zwischen den Teilnehmenden auf jeder Stufe an. Während sie auf der ersten Stufe nur mit einer oder wenigen Personen kommunizieren, nehmen ab Stufe II "sowohl die Anzahl der Kommunikationspartner als auch die Frequenz langsam zu, bis ab Stufe $V$ wieder individuelle Strategien überhand nehmen» (Salmon 2004, S. 28).

3 Die z. T. verwendeten Indizes A und B weisen darauf hin, dass die Rollen/ Aufgaben innerhalb des Konzepts verschiedenen Personen zukommen. 


\section{Literatur}

Anderson, T./Rourke, L./Garrison, D. A./Archer, W. "Assessing Teaching Presence in a Computer Conferencing Context.» Journal of Asynchronous Learning Networks 5, H. 2 (2001). 〈http://www.sloan-c.org/publications/jaln/v5n2/pdf/v5n2_anderson.pdf> (4.4.2007).

Arnold, P./Kilian, L./Thillosen, A./Zimmer, G. E-Learning - Handbuch für Hochschulen und Bildungszentren: Didaktik, Organisation, Qualität. Nürnberg: BW Bildung und Wissen Verlag und Software GmbH, 2004.

Baacke, D. «Medienkompetenz als zentrales Operationsfeld von Projekten.» Handbuch Medien: Medienkompetenz. Modelle und Projekte. Hrsg. von D. Baacke, S. Kornblum, J. Lauffer, L. Mikos u. G. A. Thiele. Bonn: Bundeszentrale für Politische Bildung, 1999. 31-35.

Czerwionka, T./de Witt, C. «Betreuung von Online-Communities of Inquiry.» eLearning-Didaktik. Hrsg. von R. Arnold u. M. Lermen. Baltmannsweiler: Schneider, 2006. 118-131. 〈http://www.fernunihagen.de/KSW/ifbm/ bildmed/pdf/deWitt_Czerwionka_Betreuung_von_Online-Cols.pdf, (4.4.2007)

Euler, D. «Selbstgesteuertes Lernen mit Multimedia und Telekommunikation gestalten.» Handbuch E-Learning. Expertenwissen aus Wissenschaft und Praxis. Hrsg. von A. Hohenstein u. K. Wilbers. Köln: Dt. Wirtschaftsdienst, 2001. Beitrag 4.1.

Garrison, D. R./Anderson, T. E-Learning in the 21st Century. A Framework for Research and Practice. London/New York: RoutledgeFalmer, 2003.

Garrison, D. R./Anderson, T./Archer, W. «Critical Inquiry in a Text-Based Environment: Computer Conferencing in Higher Education.» The Internet And Higher Education 2, H. 2/3 (2000). 87-105.

Heckman, R./Annabi, H. A Content Analytic Comparison of FTF and ALN Case-Study Discussions. Proceedings of the 36th Hawaii International Conference on System Sciences (HICSS-36). (2003). «http://csdl2.computer.org/comp/proceedings/hicss/2003/1874/01/187410003a.pdf/ (4.4.2007)

Johnson, D. W./Johnson, R. T./Stanne, M. B. Cooperative Learning Methods: A Meta-Analysis. Minneapolis: University of Minnesota, 2000. 〈http://www.co-operation.org/pages/cl-methods.html〉 (4.4.2007).

Kerres, M. Multimediale und telemediale Lernumgebungen: Konzeption und Entwicklung. München: Oldenbourg, 2001.

Kerres, M./Nübel, I./Grabe, W. «Gestaltung der Betreuung beim E-Learning.» E-Learning in Hochschulen und Bildungszentren. Hrsg. von D. Euler u. S. Seufert. München: Oldenbourg, 2005. 335-350.

Nübel, I./Kerres, M. «Splitting Tutor Roles. Supporting Online-Learners with Group Tutors and Subject Tutors.» Supporting the Learner in Distance Education and E-Learning. Proceedings of the Third EDEN Research Workshop. Hrsg. von U. Bernath u. A. Szücs. (2004). Oldenburg: Bibliotheks- und Informationssystem der Universität Oldenburg. 324-328.

Peters, O. Die Fernuniversität. Das erste Jahr. Aufbau, Aufgaben, Ausblicke. Hagen: v. d. Linnepe Verlagsgesellschaft, 1976. 
Rautenstrauch, C. Tele-Tutoren. Qualifizierungsmerkmale einer neu entstehenden Profession. Bielefeld: Bertelsmann, 2001.

Reinmann-Rothmeier, G./Mandl, H. «Analyse und Förderung kooperativen Lernens in netzbasierten Umgebungen.» Zeitschrift für Entwicklungspsychologie und Pädagogische Psychologie 34, H. 1 (2002). 44-57.

Rourke, L./Anderson, T. «Using Peer Teams to Lead Online Discussions.» Journal of Interactive Media in Education 7, H. 1 (2002). «http://wwwjime.open.ac.uk/2002/1/> (4.4.2007).

Salmon, G. E-tivities - Der Schlüssel zu aktivem Online-Lernen. Zürich: Orell Füssli Verlag AG, 2004.

Schenk, B. "Moderation.» CSCL-Kompendium. Hrsg. von J. M. Haake, G. Schwabe u. M. Wessner. München: Oldenbourg, 2004. 215-225.

Schröder, R./Wankelmann, D. Theoretische Fundierung einer e-LearningDidaktik und der Qualifizierung von e-Tutoren im Rahmen des Leonardo-Projekts e-Tutor - Entwicklung einer europäischen e-Learning-Didaktik. Paderborn: Universität Paderborn, 2002.

Thomaschewski, J. "Die mentorielle Betreuung im Online-Studium.» LIMPACT, Sonderausgabe VFH (2005). 45-48. 〈http://www.bibb.de/dokumente/pdf/limpact_vfh.pdf> (4.4.2007)

de Witt, C./Czerwionka, T./Mengel, S. Mentorielle Betreuung an der FernUniversität in Hagen. Rückblicke, Einblicke, Ausblicke. Hagen: FernUniversität in Hagen, Institut für Bildungswissenschaft und Medienforschung, 2006. 